\title{
A Case Report on Periapical Abscess with Attrition and Abrasion of Maxillary Anteriors
}

\section{Dr Fatima Zuhara Shaikh*}

Clinical Practitioner at South Mumbai, India

*Corresponding Author: Dr Fatima Zuhara Shaikh, Clinical Practitioner at South Mumbai, India.

Received: October 14, 2019; Published: October 28, 2019

DOI: $10.31080 /$ ASDS.2019.03.0677

\begin{abstract}
The aim of this study was to evaluate the success rate of endodontic treatment. In the reported case, conventional endodontic therapy was successful and sufficient enough to eliminate the periapical infection allowing periapical bone formation and absence of symptomatology.This report presents the successful management of an endodontic lesion in maxillary anterior teeth using conventional endodontic therapy.

Keywords: Endodontic Treatment; Attrition
\end{abstract}

\section{Introduction}

The ultimate aim of endodontic treatment is to preserve the tooth in the oral cavity and prevent the recurrence of infection or treat the periapical abscess. Evaluation is required by taking proper radiograph and accurate treatment plan to be made [1-3].

\section{Case}

A 55-years old female reported to Clinic with a chief complaint of pain and sensitivity on upper front region of the jaw since past 10days.

\section{Medical history}

No relevant medical history

Diagnosis

Clinical examination reveals Abrasion and Attrition in maxillary right central and lateral and left central incisor. The tooth was tender on percussion.

Radiographically there was abscess and external resorption related to teeth \#11 1221.

A Diagnosis of chronic irreversible pulpitis was established and a treatment plan of Root Canal was planned. Patient was explained the procedure.

\section{Treatment plan}

\section{$1^{\text {st }}$ sitting}

- With informed consent to make the procedure painless and smooth local anaesthesia was given
- $2 \%$ Lignocaine with adrenaline

- Root canal opening was done

- The working length was determined by electronic apex locator and confirmed with radiograph (since there is an abscess the tactile sensation was difficult to locate the anatomical apex)

- $\quad$ Biomechanical preparation was done using Healix Conmem M3 system.

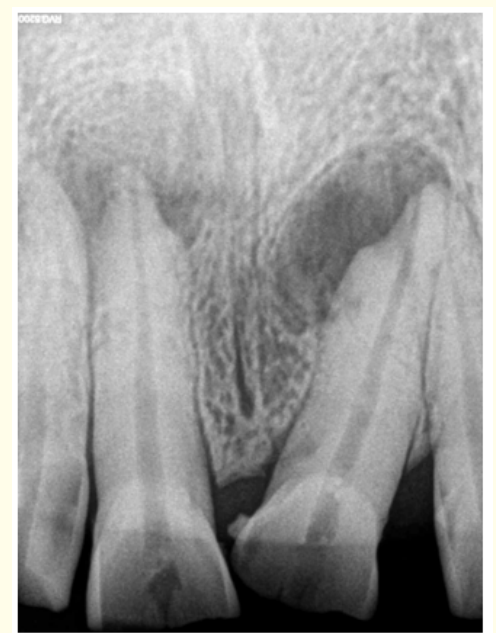

Figure 1: Pre-operative radiograph of teeth \# 111221 showing periapical abscess and external resorption. 

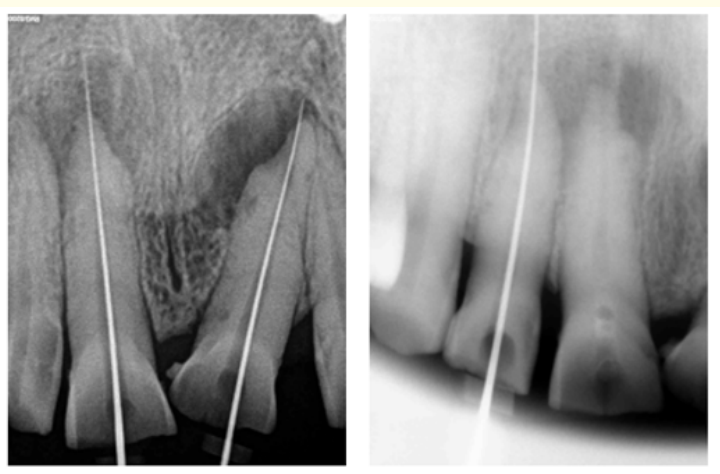

Figure 2: Working length radiograph of teeth \# 111221.

\section{$2^{\text {nd }}$ Sitting}

- Irrigation done with standardised irrigation protocol. (3\% sodium hypochlorite and normal saline)

- $\quad 17 \%$ Ethylene-diamine-tetra-acêable acíd (EDTA) used for shaping the canal.

- Metapex dressing was given to reduce the periapical infection and was pushed into the lesion. Patient was recalled after 14 days.
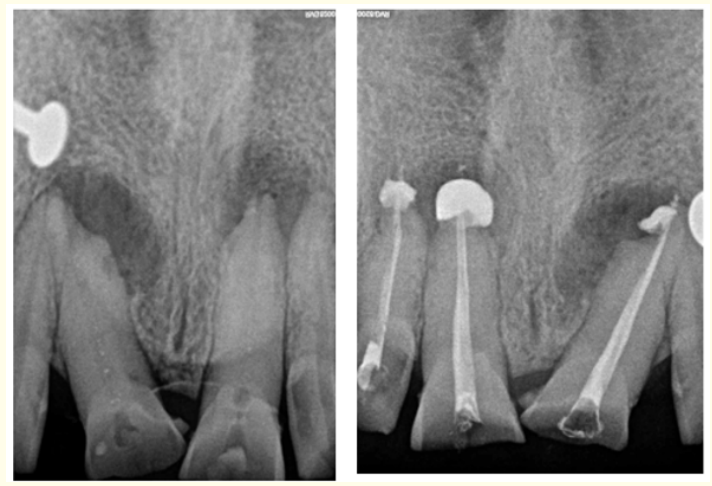

Figure 3: Calcium hydroxide dressing given and Metapex dressing followed beyond apex in periapical abscess.

$3^{\text {rd }}$ Sitting

- Metapex dressing was removed only from the canals as left in the periapical area.

- Obturation was done with cold lateral condensation with resin based cement (AH plus sealer)

- Post obturation restoration done with composite material.
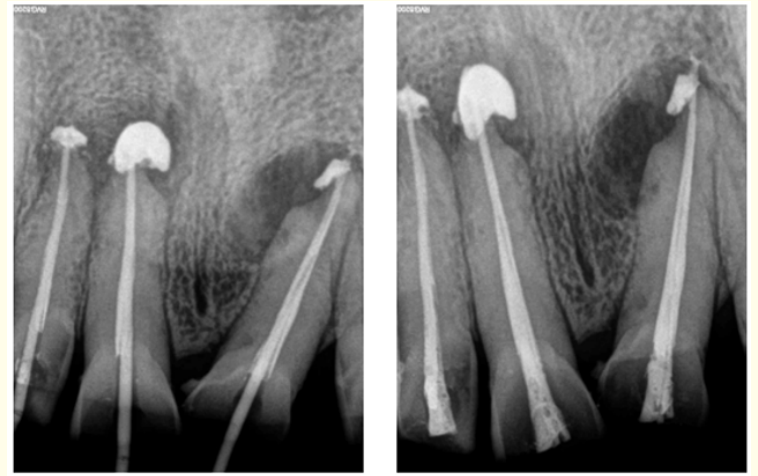

Figure 4: Master come radiograph of teeth 111221 and After the accessory gutta percha placed. (Periapical area shows metapex dressing).

The case details are mentioned below

\begin{tabular}{|l|c|c|}
\hline $\begin{array}{l}\text { Tooth } \\
\text { No }\end{array}$ & $\begin{array}{c}\text { Working } \\
\text { Length }\end{array}$ & $\begin{array}{c}\text { Master } \\
\text { Cone }\end{array}$ \\
\hline 11 & $17 \mathrm{MM}$ & $\# 304 \%$ \\
\hline 12 & $18 \mathrm{MM}$ & $\# 304 \%$ \\
\hline 21 & $18 \mathrm{MM}$ & $\# 304 \%$ \\
\hline
\end{tabular}

Table 1

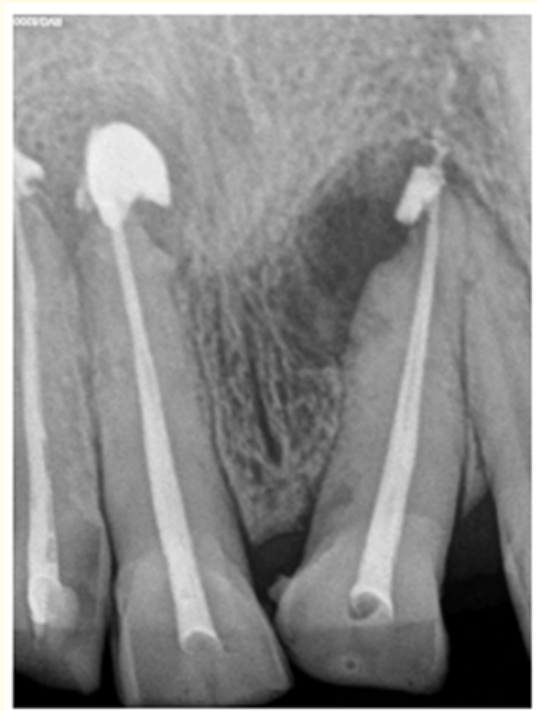

Figure 5: Post-operative radiograph of teeth \#11 1221. 


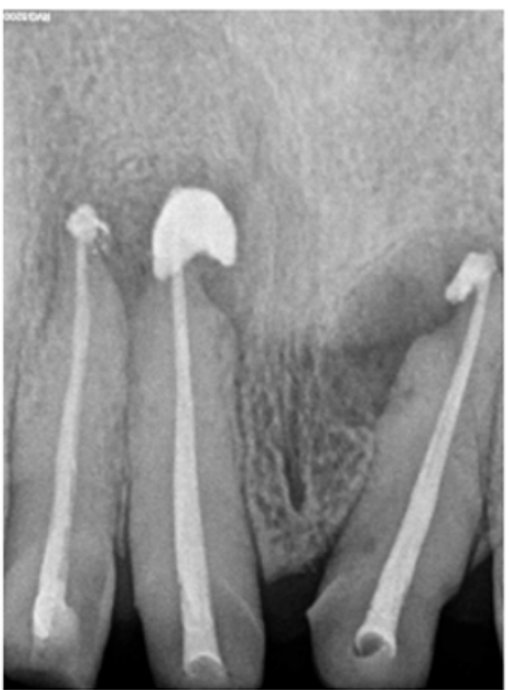

Figure 6: Follow up after 15days-Healing seen.

\section{Conclusion}

Endodontic treatment in such case require a knowledge of dental anatomy and variations with radiographic evaluation. A complete healing of the lesion will be visible after 6 months follow up of the same.

\section{Bibliography}

1. Aidin Sooratgar., et al. "Management of an Endodontic-Periodontal lesion of Maxillary lateral incisor with palatal radicular groove-a case report". IEJ 11.2 (2016): 142-145.

2. Airton Oliveira Santos-Juniour., et al. "Success or failure of Endodontic treatment-Retrospective study". Journal of Conservative Dentistry (JCD) 22 (2019).

3. Solely Arslan., et al. "Efficacy of different endodontic irrigation protocols on shear bond strength to coronal Dentin". Journal of Conservative Dentistry (JCD) 22 (2019): 223-227.

\section{Volume 3 Issue 11 November 2019}

(C) All rights are reserved by Fatima Zuhara Shaikh. 